\title{
Retrospective study on the efficacy of bisphosphonates in tyrosine kinase inhibitor-treated patients with non-small cell lung cancer exhibiting bone metastasis
}

\author{
XIAOXIAN CUI*, SHAOLI LI*, JINCUI GU*, ZIYING LIN, BIPENG LAI, \\ LIXIA HUANG, JINLUN FENG, BAOMO LIU and YANBIN ZHOU
}

Department of Respiratory Medicine, The First Affiliated Hospital of Sun Yat-sen University,
Guangzhou, Guangdong 510080, P.R. China

Received August 19, 2018; Accepted August 14, 2019

DOI: 10.3892/ol.2019.10870

\begin{abstract}
Bisphosphonates (Bps) inhibit the maturation of osteoclasts and suppress the adhesion of cancer cells to the bone matrix. They are recommended as the standard treatment for tumors exhibiting bone metastasis (BM). However, whether Bps can improve the prognosis of patients with tyrosine kinase inhibitor (TKI)-treated non-small cell lung cancer (NSCLC) exhibiting BM remains unclear. A total of 129 patients with NSCLC initially diagnosed with BM at The First Affiliated Hospital of Sun Yat-Sen University (Guangzhou, China) between January 2005 and December 2017 were analyzed in the present retrospective study. Median progression free survival (mPFS) time, median bone metastasis overall survival (mBM-OS) time and bone-associated progression-free survival were analyzed. Among the 129 patients, patients treated with Bps experienced significantly prolonged PFS time [mPFS: 7.1 vs. 5.1 months; hazard ratio (HR), 0.51; confidence interval $(\mathrm{CI}), 0.30-0.87 ; \mathrm{P}=0.0114]$ in comparison with patients not treated with Bps. Of the 49 patients treated with frontline TKIs (EGFR TKIs or ALK TKI), 32 received Bps at the same time, while 17 patients received TKIs alone. The results revealed that mPFS time was significantly greater in the TKIs plus Bps group than in the TKIs alone group (mPFS: 11.2 vs. 6.9 months; HR, 0.13; CI, 0.05-0.35; P<0.0001). Significantly prolonged BM-OS time was also observed in the combination group in comparison with the TKIs alone group (mBM-OS: 31 vs. 22 months; HR, 0.31; CI, 0.10-0.96; $\mathrm{P}=0.0413$ ). The
\end{abstract}

Correspondence to: Professor Yanbin Zhou, Department of Respiratory Medicine, The First Affiliated Hospital of Sun Yat-sen University, 58 Zhongshan Road, Guangzhou, Guangdong 510080, P.R. China

E-mail: sysuzyb@aliyun.com

${ }^{*}$ Contributed equally

Key words: non-small cell lung carcinoma, bone metastasis, bisphosphonate, prognosis, tyrosine kinase inhibitors present study demonstrated that among the patients who received TKIs (EGFR TKIs or ALK TKIs), those who also received Bps experienced significantly longer PFS time and tended to exhibit significantly improved BM-OS time, which indicated that Bps should be added to the treatment regimen of patients with NSCLC exhibiting genetic mutations and bone metastasis who have been prescribed TKIs (EGFR TKIs or ALK TKIs).

\section{Introduction}

Lung cancer is a leading cause of morbidity and mortality in China, and it is the most common form of cancer in the country (1). In 2015, lung cancer affected 733.3 per 100,000 people in China, with an overall mortality rate of 177.8 per 100,000 (1). Non-small cell lung cancer (NSCLC) accounts for $80-85 \%$ percent of lung cancer diagnoses (2), and the majority of patients with NSCLC remain undiagnosed until the disease had progressed to a late stage (3). The 5-year survival rate for patients with stage IV lung cancer is $<5 \%$ (4). Bone metastasis (BM) is a common complication of these advanced tumors, and it is closely associated with lung cancer-associated mortality (5). Of the patients with lung cancer, 22-60\% develop minor metastases in the bone marrow (6). Of the patients with NSCLC, 30-40\% develop BM and have a median survival time of only $\sim 6$ months (7). Thus, a study regarding the prevention and treatment of BM in patients with NSCLC may significantly improve overall survival (OS) time.

Zoledronate is a third-generation heterocyclic bisphosphonates (Bps) that can effectively treat BM-associated complications, such as pathological fractures, hypercalcemia, spinal cord compression and intractable pain (8). It is the standard of care treatment for tumors with BM as it can inhibit the maturation of osteoclasts, thereby affecting their activity and biological function (8). Zoledronate can also induce apoptosis in osteoclasts by firmly adsorbing on to the surface of bone trabecula, preventing bone dissolution and suppressing the adhesion of cancer cells on the bone matrix (9). Preclinical studies have demonstrated the antitumor activity of Zoledronate, as it can inhibit the proliferation, invasion, metastasis and angiogenesis of NSCLC cells $(10,11)$. Notably, 
preclinical studies have also demonstrated that zoledronate can enhance the antitumor effect of gefitinib in patients with epidermal growth factor receptor (EGFR)-mutant NSCLC. In 2015, a retrospective study of 62 patients with NSCLC exhibiting $\mathrm{BM}$ demonstrated that significantly prolonged progression-free survival (PFS) time and OS time were achieved in patients treated with a combination of EGFR TKI and Bps in comparison with patients treated with EGFR TKI monotherapy [median (m)PFS: 15.0 vs. 7.3 months; $\mathrm{P}=0.03$ and OS, 25.2 vs. 10.4 months; $\mathrm{P}=0.0015]$ (12). Another retrospective study that included 356 patients with NSCLC exhibiting $\mathrm{BM}$ reported that in comparison with EGFR TKI monotherapy, EGFR TKI in combination with Bps demonstrated longer PFS time [11.6 vs. 9.3 months; hazard ratio (HR), 0.68; $\mathrm{P}=0.009$ ] (13). However, in comparison with the previously discussed retrospective study, contradictory results of OS time were observed in that no significant difference was observed in OS between the study group and the control group (20.5 vs. 19.5 months; HR, 0.95; $\mathrm{P}=0.743$ ) (13).

Therefore, in order to assess whether Bps can prolong the OS time of patients with NSCLC exhibiting BM that were treated with EGFR TKIs, the present study analyzed the prognosis and impact of zoledronate on prognosis in 129 patients with NSCLC exhibiting BM. The present study also investigated the effect of Bps therapy on bone lesion progression in patients with NSCLC treated with EGFR TKIs.

\section{Materials and methods}

Patients. A total of 129 patients with NSCLC who were initially diagnosed with BM at The First Affiliated Hospital of Sun Yat-Sen University (Guangzhou, China) from January 2005 to December 2017 were analyzed in the present retrospective study. The following inclusion criteria were used: i) Pathological or cytological diagnosis of NSCLC; ii) willing and able to complete whole-body evaluation (including CT, ECT, PET-CT, abdominal b-ultrasound and MRI); iii) at least one BM was observed upon the initial diagnosis; and iv) standard treatment and regular systemic assessment. The following exclusion criteria were used: i) Secondary malignancy with $\mathrm{BM}$ of unknown origin; ii) participation in other clinical trials involving bisphosphonate analogues; and (iii) no BM observed at the time of initial diagnosis, or BM occurred after standard treatment. The present study was approved by the Ethics Committee of The First Affiliated Hospital of Sun Yat-sen University. A waiver was granted excusing the requirement of written informed consent due to the retrospective nature of the present study.

Baseline clinical pathology data. All information obtained on the patients were acquired from The First Affiliated Hospital of Sun Yat-Sen University hospital by searching the medical records of patients with NSCLC. The present study assessed age, sex, Eastern Cooperative Oncology Group (ECOG) performance status (PS), smoking status, pathological type, genetic mutations, the number of BMs, weight-bearing BMs, skeleton-associated events (SAEs), presence or absence of brain metastases, lactate dehydrogenase (LDH) levels, alkaline phosphatase (ALP) levels, median Bps time, median Bps duration, and treatment. All patients were treated according to NCCN guidelines (14). All patients received tyrosine kinase inhibitor as the first-line treatment. EGFR TKIs, which include gefitinib, erlotinib, and icotinib, were given to the patients with sensitive EGFR mutations. Anaplastic lymphoma kinase (ALK) TKI, referred to as crizotinib, was given to the patients harboring ALK rearrangements. When the disease progressed, platinum plus 1 third-generation chemotherapy regime was administered as the second-line treatment. Another third-generation chemotherapy regime was prescribed when the disease progressed on the second-line treatment. There is no standard of care beyond third-line treatment. The third-generation chemotherapy drugs include docetacel, gemcitabine, paclitaxel, vinorelbine, and pemetrexed.

Follow up information. Participants underwent whole-body evaluations every 3 months after diagnosis of NSCLC with BM. The present study searched inpatient and outpatient care inspection results in the hospital information system, telephone follow-up treatment and associated sources for information on prognosis. Follow-up time was defined as the time from diagnosis of NSCLC with BM and first treatment to death or April 1, 2018. PFS was defined as the time from the initial treatment to progression of, or death from, the disease. BM-OS time was defined as the time from BM to death. Bone-associated PFS time (BaPFS) was defined as the time from the first treatment to advancement of bone lesions, which was evaluated following radiographic assessment by CT, MRI, X-ray, PET-CT scans, or radionuclide bone scan, and comparison with the baseline bone damage. Advancement of bone lesions included the development of at least one new BM, or at least $25 \%$ increase in one bone lesion (15). All endpoints were assessed according to the Response Evaluation Criteria in Solid Tumor (RECIST 1.1) (16).

Statistical analysis. SPSS software (version 20.0; SPSS, Inc.) was used for the statistical analysis. The Kaplan-Meier method was used for the survival analysis, and the log-rank test was used to compare different groups. A Cox proportional risk regression model was used to analyze univariate and multivariate variants. Univariate variants with $\mathrm{P}<0.2$ were selected for the multivariate variant analysis. $\mathrm{P}<0.05$ was considered to indicate a statistically significant difference. One researcher independently analyzed the data and two other researchers reanalyzed the data twice.

\section{Results}

Patient clinical characteristics. A total of 129 patients with NSCLC who were initially diagnosed with BM at The First Affiliated Hospital of Sun Yat-Sen University between January 2005 and December 2017 were analyzed in the present retrospective study. Among these, 94 patients underwent genetic analysis before treatment: EGFR mutations were detected in 50 patients, 9 patients were observed to possess ALK mutations, and 2 patients were observed to possess ROS1 mutations. Among the 50 patients with EGFR mutations, 24 consented to receive combination treatment of EGFR TKIs and Bps, and 14 patients received EGFR TKIs monotherapy. Among patients with wild-type EGFR, 2 patients were given the EGFR TKIs plus Bps regimen. In addition, 5 patients 


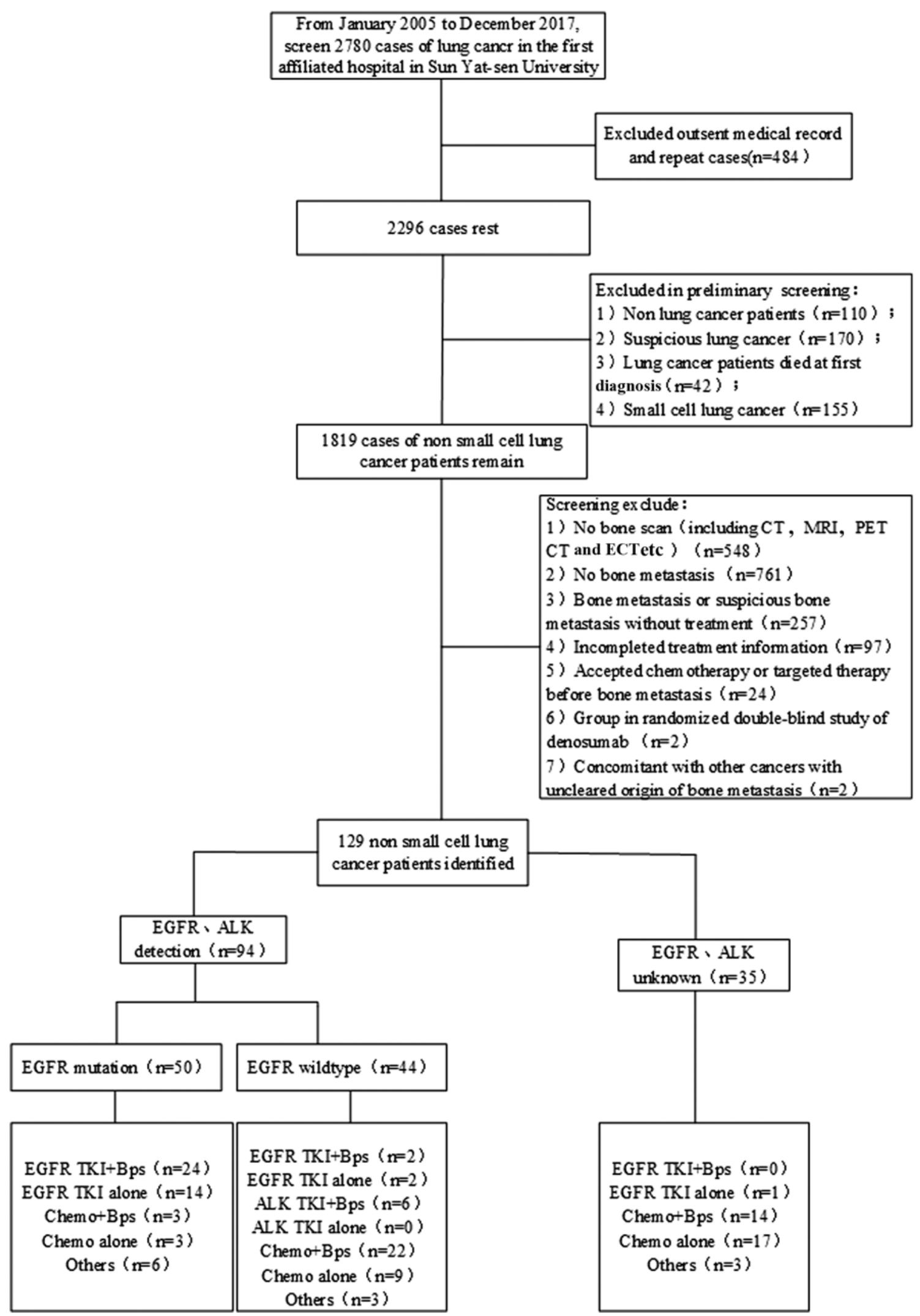

Figure 1. Flow chart of case screening. EGFR TKI, epidermal growth factor receptor tyrosine kinase inhibitor; ALK TKI, anaplastic lymphoma kinase tyrosine kinase inhibitor; chemo, chemotherapy; Bps, bisphosphonates; Others, combination therapy of TKIs and chemotherapy.

with ALK mutations and 1 patient with a ROS1 mutation received ALK TKI combined with Bps. Therefore, a total of 32 patients were treated with TKIs plus Bps (Fig. 1). The median age of the patients was 55.9 years (range, 21-83 years).
Adenocarcinoma accounted for $89.14 \%$ of the lung cancer subtypes, $88.37 \%$ of the patients had ECOG PS <2, $82.17 \%$ patients had weight-bearing BM and $79.84 \%$ of the patients had BM at 2 or more sites (Table I). 
Table I. Demographic and baseline clinical characteristics of the patients.

\begin{tabular}{|c|c|c|c|}
\hline Patient characteristic & No. of patients $(n=129)$ & TKI+Bps $(n=32)$ & TKI alone $(n=17)$ \\
\hline Median age, years (range) & $55.9(21-83)$ & $56.9(21-83)$ & $58(28-78)$ \\
\hline \multicolumn{4}{|l|}{ Sex, $n$} \\
\hline Male & 82 & 18 & 8 \\
\hline Female & 47 & 14 & 9 \\
\hline \multicolumn{4}{|l|}{ ECOG performance status, $n$} \\
\hline $0-1$ & 114 & 29 & 15 \\
\hline$\geq 2$ & 15 & 3 & 2 \\
\hline \multicolumn{4}{|l|}{ Smoking history, $\mathrm{n}$} \\
\hline Yes or ever & 53 & 10 & 5 \\
\hline No & 76 & 22 & 12 \\
\hline \multicolumn{4}{|l|}{ Pathological type, $n$} \\
\hline Adenocarcinoma & 115 & 29 & 17 \\
\hline Non-adenocarcinoma & 14 & 3 & 0 \\
\hline \multicolumn{4}{|l|}{ Gene mutation, $\mathrm{n}$} \\
\hline EGFR mutation & 50 & 24 & 14 \\
\hline L858R & 17 & 8 & 2 \\
\hline 19DEL & 25 & 12 & 10 \\
\hline Rare mutation & 7 & 4 & 1 \\
\hline Unknown & 35 & 0 & 1 \\
\hline ALK mutation & 9 & 5 & 1 \\
\hline ROS1 mutation & 2 & 1 & 1 \\
\hline \multicolumn{4}{|l|}{ Brain metastasis, $\mathrm{n}$} \\
\hline Yes & 32 & 12 & 4 \\
\hline No & 97 & 20 & 13 \\
\hline \multicolumn{4}{|l|}{ Weight-bearing bone metastasis, $\mathrm{n}$} \\
\hline Yes & 106 & 26 & 14 \\
\hline No & 23 & 6 & 3 \\
\hline \multicolumn{4}{|l|}{ Bone metastasis number, $\mathrm{n}$} \\
\hline Single (1) & 26 & 6 & 2 \\
\hline Multiple ( $\geq 2$ ) & 103 & 26 & 15 \\
\hline \multicolumn{4}{|l|}{ Skeletal related events, $\mathrm{n}$} \\
\hline Yes & 23 & 10 & 2 \\
\hline No & 106 & 22 & 15 \\
\hline \multicolumn{4}{|c|}{ Total number of bisphosphonates agents, $n$} \\
\hline $1-2$ & 26 & 12 & 0 \\
\hline $3-6$ & 40 & 12 & 0 \\
\hline$>6$ & 13 & 8 & 0 \\
\hline Median Bps time, days, (range) & $40.72(0-609)$ & $52.34(0-609)$ & - \\
\hline Median Bps duration, days (range) & $84.75(21-846)$ & $106.86(24-846)$ & - \\
\hline $\mathrm{LDH}>300 \mathrm{IU} / 1, \mathrm{n}$ & 22 & 5 & 4 \\
\hline ALP>60 IU/l, b & 16 & 5 & 3 \\
\hline
\end{tabular}

TKI, tyrosine kinase inhibitors; Bps, bisphosphonates ECOG, Eastern Cooperative Oncology Group; EGFR, epidermal growth factor receptor; LDH, lactic dehydrogenase; ALP, alkaline phosphatase.

PFS time. In all 129 patients, in comparison with the non-Bps regimen group, patients in the group whose treatment regimen included Bps had significantly prolonged PFS time [mPFS, 7.1 vs. 5.1 months; hazard ratio (HR), 0.51; confidence interval (CI) $0.30-0.87$; $\mathrm{P}=0.0114$; Fig. 2A]. Of the 49 patients who were treated with TKIs, 32 of them received TKIs (EGFR TKIs or ALK TKI) plus Bps, while 17 patients were treated with TKIs alone. The results revealed that the median PFS time was significantly improved in the TKIs plus Bps group in comparison with the TKIs alone group (mPFS: 11.2 vs. 

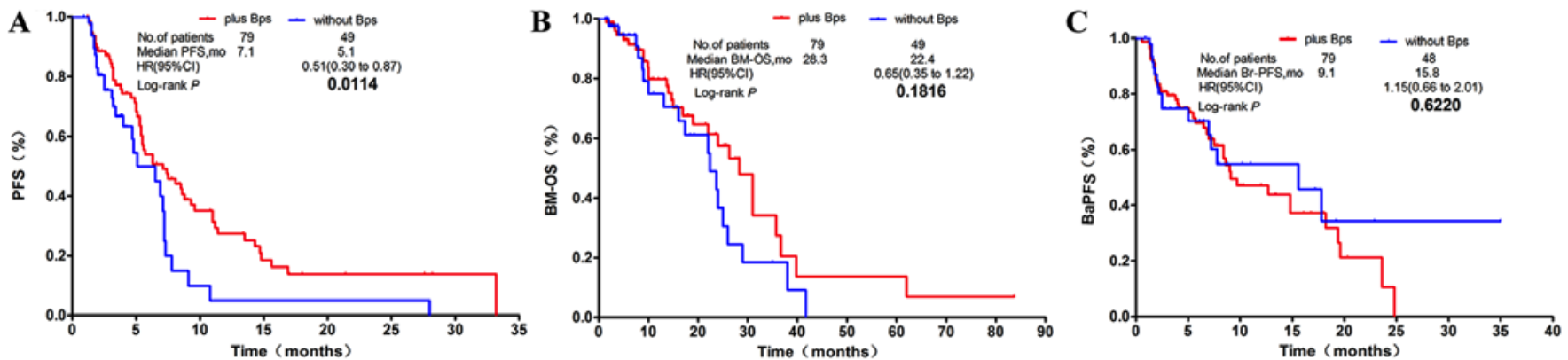

Figure 2. Kaplan-Meier survival curves of PFS, BM-OS and BaPFS time in all patients with NSCLC exhibiting BM. (A) The Bps regimen significantly prolonged PFS time over other regimens, but results were similar to (B) BM-OS and (C) BaPFS time compared with those without Bps regimen group in patients with NSCLC exhibiting BM. PFS, progression-free survival; BM-OS, bone metastasis-overall survival; BaPFS, bone-associated progression-free survival; NSCLC, non-small cell lung cancer; BM, bone metastasis; Bps, bisphosphonates; HR, hazard ratio; CI, confidence interval; mo, months.
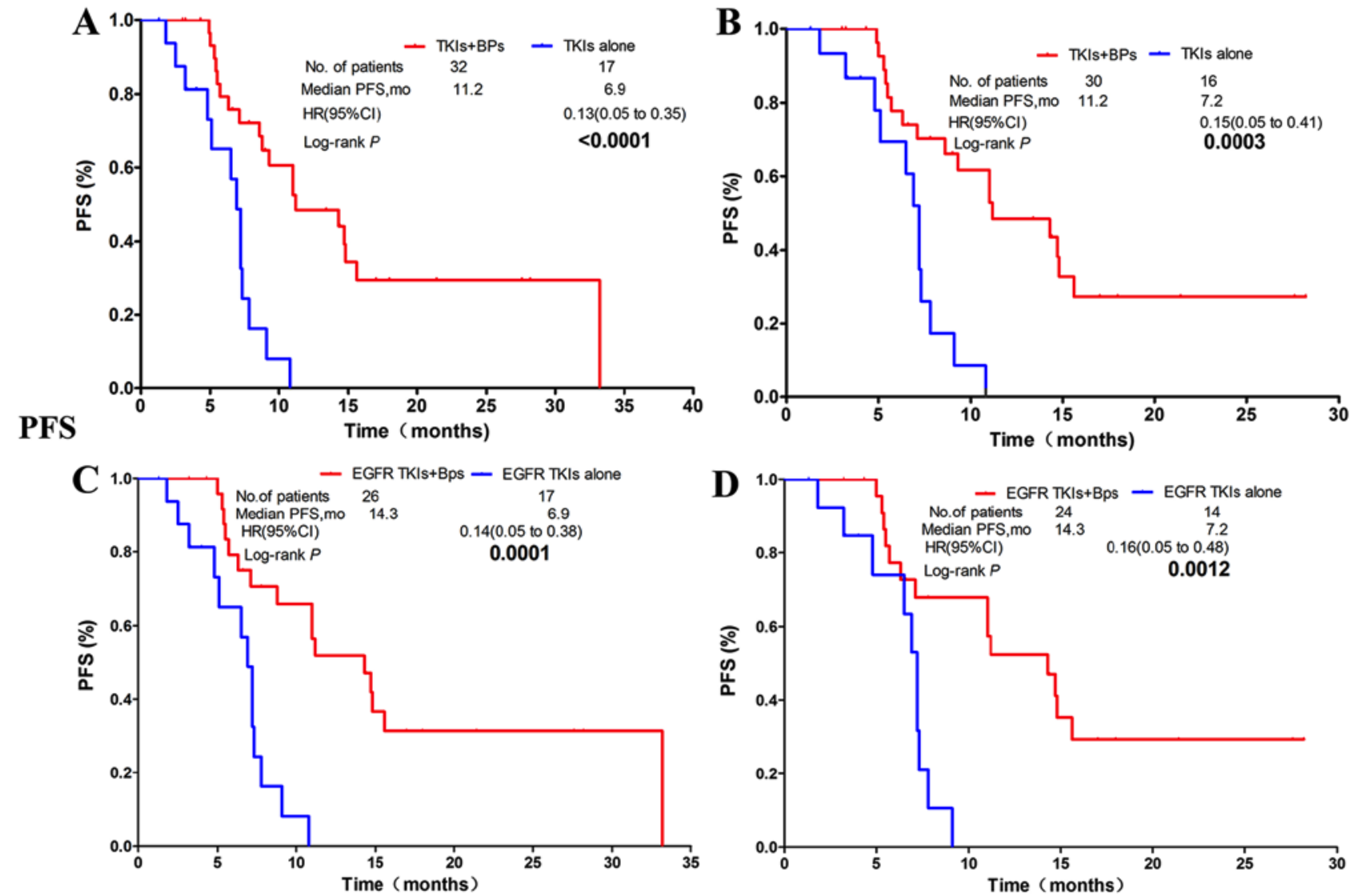

Figure 3. NSCLC patients with BM who receive TKIs and Bps had significantly better PFS time than those receiving TKIs alone. (A) NSCLC patients receiving TKIs regardless of gene mutation status. (B) NSCLC patients with EGFR mutation or ALK rearrangements. (C) NSCLC patients receiving EGFR-TKI with/without EGFR mutation. (D) NSCLC patients with sensitive EGFR mutation. NSCLC, non-small cell lung carcinoma; BM, bone metastasis; TKI, tyrosine kinase inhibitor; EGFR, epidermal growth factor receptor; PFS, progression-free survival; Bps, bisphosphonates; HR, hazard ratio; CI, confidence interval; mo, months.

6.9 months; HR, 0.13; CI, 0.05-0.35; P<0.0001; Fig. 3A). In patients harboring EGFR or ALK mutations, PFS time in the combination group was significantly longer than in patients treated with TKIs alone (mPFS: 11.2 vs. 7.2 months; HR, 0.15; CI, 0.05-0.41; P=0.0003; Fig. 3B). The EGFR TKIs plus Bps group also had a significantly longer PFS time than the EGFR TKIs alone group (mPFS: 14.3 vs. 6.9 months; HR, 0.14; CI, 0.05-0.38; P=0.0001; Fig. 3C). Significantly longer PFS time was also observed in patients with EGFR mutant NSCLC who were treated with EGFR TKIs plus Bps in comparison with those treated only with EGFR TKIs (mPFS: 14.3 vs. 7.2 months; HR, 0.16; CI, 0.05-0.48; P=0.0012; Fig. 3D).

BM-OS time. The BM-OS time of patients in the combination group was 6 months longer than that of patients in the non-Bps group. However, no statistically significant difference was observed between the two groups (mBM-OS, 28.3 vs. 22.4 months; HR, 0.65; CI, 0.35-1.22; P=0.1816; Fig. 2B). In patients who underwent TKI treatment, the combination group exhibited significantly improved BM-OS time compared with 
A

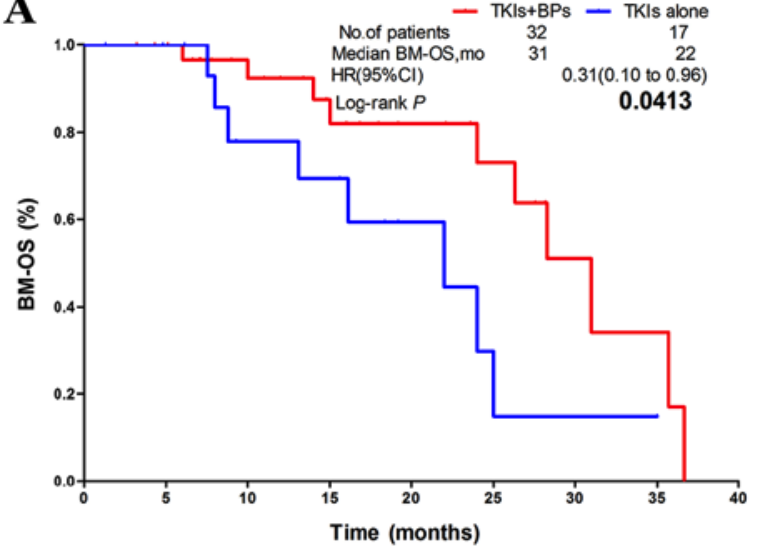

BM-OS

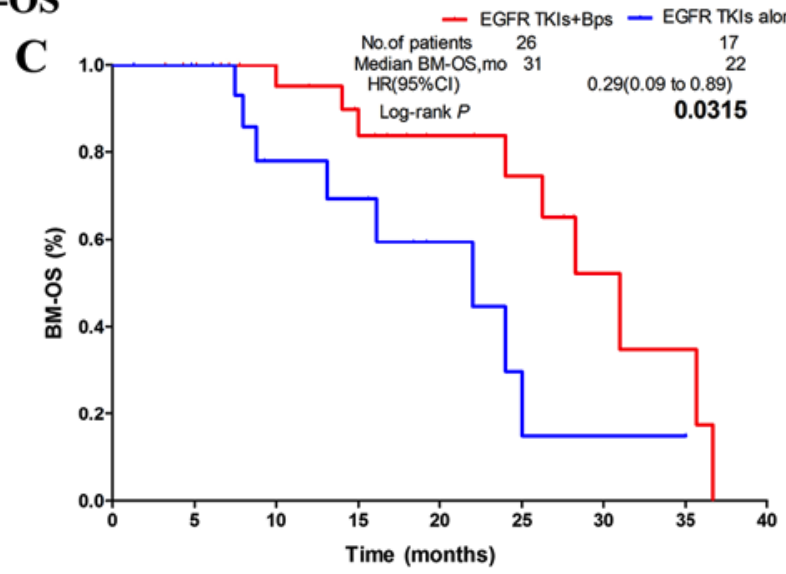

B

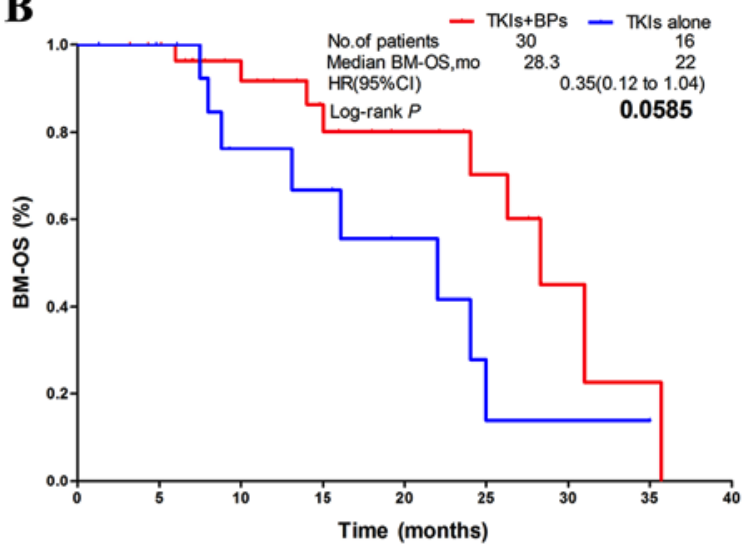

D

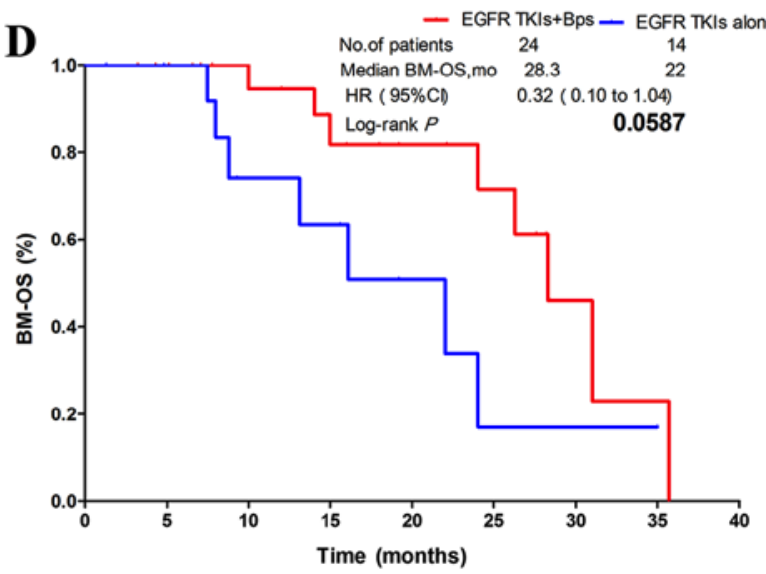

Figure 4. Combination therapy with Bps significantly prolonged BM-OS time over non-Bps groups in subgroups A and C, and meaningful trends toward statistically significant differences were observed between the combination group and non-Bps group in BM-OS in groups B and D. (A) NSCLC patients receiving TKIs regardless of gene mutation status. (B) NSCLC patients with EGFR mutation or ALK rearrangements. (C) NSCLC patients receiving EGFR-TKI with/without EGFR mutation. (D) NSCLC patients with sensitive EGFR mutation. NSCLC, non-small cell lung carcinoma; Bps, bisphosphonates; BM-OS, bone metastasis-overall survival; TKI, tyrosine kinase inhibitor; EGFR, epidermal growth factor receptor; HR, hazard ratio; CI, confidence interval; mo, months.

patients treated with TKIs alone (mBM-OS: 31 vs. 22 months; HR, 0.31; CI, 0.10-0.96; P=0.0413; Fig. 4A). However, the subgroup of patients with EGFR or ALK mutations exhibited no statistically significant difference in BM-OS time between the combination group and the TKIs alone group (mBM-OS: 28.3 vs. 22.0 months; HR, 0.35; CI, 0.12-1.04; $\mathrm{P}=0.0585$; Fig. 4B). In patients treated with EGFR TKIs, the combination group exhibited significantly improved BM-OS time in comparison with the EGFR TKIs alone group (mBM-OS: 31 vs. 22 months; HR, 0.29; CI, 0.09-0.89; P=0.0315; Fig. 4C). In patients with EGFR mutations who underwent EGFR TKIs treatment, no statistically significant difference in BM-OS time was observed between the combination group and the EGFR TKIs alone group (mBM-OS: 28.3 vs. 22.0 months; HR, 0.32; CI, 0.10-1.04; P=0.0587; Fig. 4D).

BaPFS time. No statistically significant difference was observed between the Bps-treated group and the non-Bpstreated group (mBaPFS: 9.1 vs. 15.8 months; HR, 1.15; CI, 0.66-2.01; $\mathrm{P}=0.6220$; Fig. $2 \mathrm{C}$ ). The subgroup analysis revealed that, in patients who received TKIs, there was also no statistically significant difference in $\mathrm{mBaPFS}$ time between the Bps-treated and the non-Bps-treated group (mBaPFS: 18.2 vs. 17.8 months; HR, 0.63; CI, 0.23-1.74; P=0.3731; Fig. 5A).
Bps treatment also had no significant effect on mBaPFS time in patients with EGFR or ALK mutant NSCLC treated with TKIs (mBaPFS, 14.8 vs. 7.8 months; HR, 0.72; CI, 0.23-2.07; $\mathrm{P}=0.5367$; Fig. 5B). Similar innocuous trends were observed in patients treated with EGFR TKIs (mBaPFS: 18.2 vs. 17.8 months; HR, 0.61; CI, 0.22-1.73; $\mathrm{P}=0.3507$; Fig. 5C), and in patients with EGFR mutant NSCLC who received EGFR TKI therapy (mBaPFS: 14.8 vs. 7.8 months; HR, 0.56; CI, 0.18-1.74; $\mathrm{P}=0.3143$; Fig. 5D).

Univariate and multivariate analysis of BM-OS time and BaPFS time. As presented in Table II, the results of the univariate analysis in all 129 patients with NSCLC exhibiting BM demonstrated that the BM-OS time prognosis of female patients was significantly better than that of male patients (BM-OS: HR, 0.44; CI, 0.22-0.87; P=0.018). However, being female was revealed to be a prognostic risk factor for BaPFS time (BaPFS: HR, 1.89; CI, 1.12-3.18; $\mathrm{P}=0.018$ ). The non-SAEs group had a significantly longer BM-OS time than the SAE group (HR, 3.21; CI, 1.13-9.15; $\mathrm{P}=0.029$ ). The ALP<160 IU/1 group had significantly improved BM-OS time in comparison with the ALP $\geq 160$ IU/1 group (HR, 3.02; CI, 1.43-6.40; $\mathrm{P}=0.004)$. Patients older than 55.9 years had longer BaPFS time compared with that in younger patients (HR, 0.97; CI, 

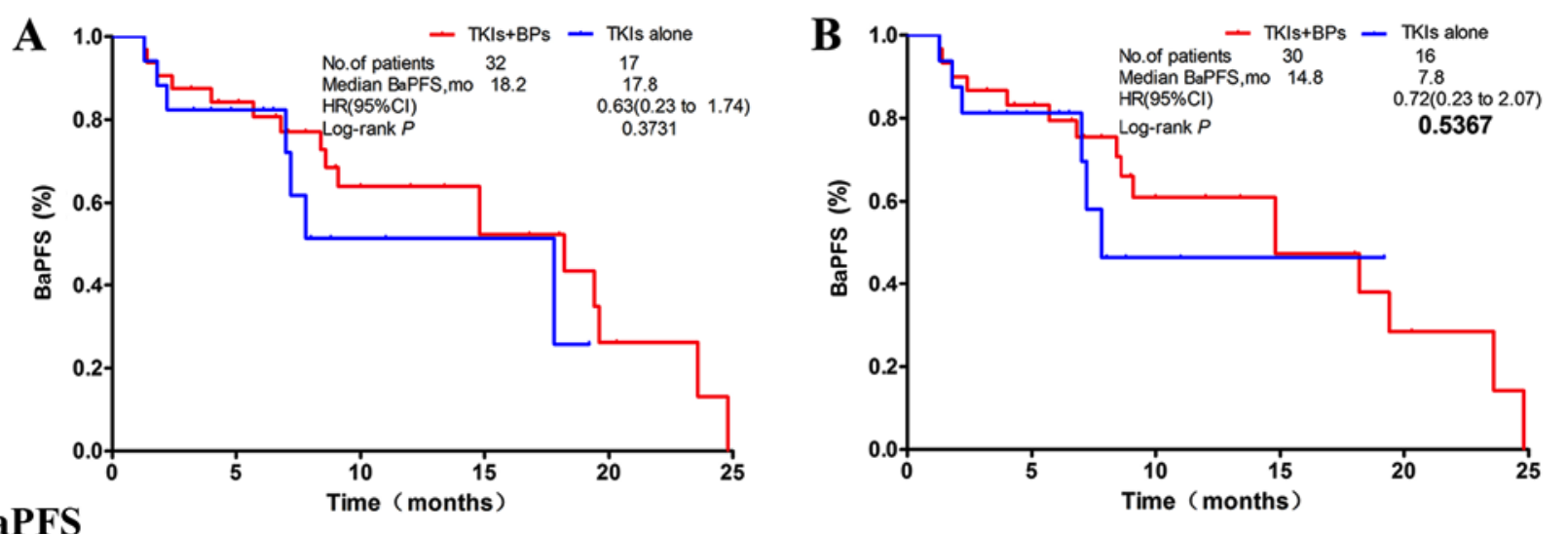

BaPFS
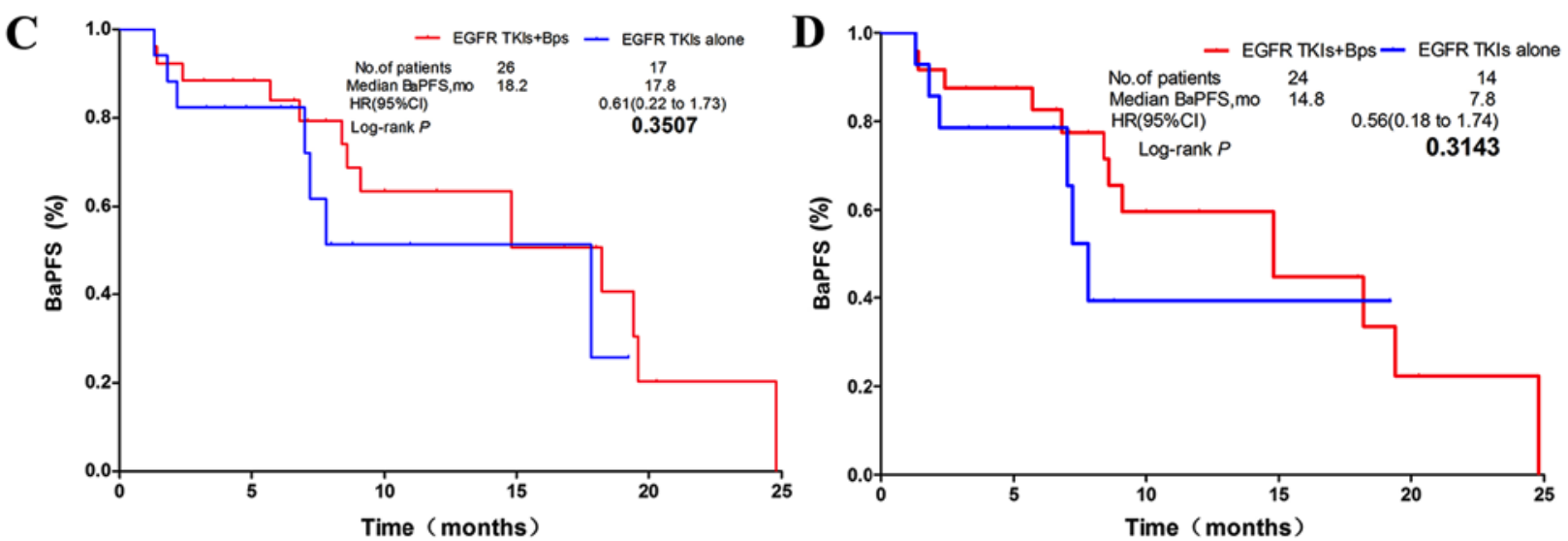

Figure 5. Regardless of the type of gene mutation, no statistically significant differences were observed in BaPFS time between the combination regimen and the non-Bps regimen in groups A, B, C, and D. (A) NSCLC patients receiving TKIs regardless of gene mutation status. (B) NSCLC patients with EGFR mutation or ALK rearrangements. (C) NSCLC patients receiving EGFR-TKI with/without EGFR mutation. (D) NSCLC patients with sensitive EGFR mutation. NSCLC, non-small cell lung carcinoma. HR, hazard ratio; TKI, tyrosine kinase inhibitor; EGFR, epidermal growth factor receptor; CI, confidence interval; mo, months; bone-associated PFS time.

0.95-0.99; $\mathrm{P}=0.009)$. $\mathrm{BMN} \geq 2$ was a risk factor for prognosis of BaPFS time (HR, 2.37; CI, 1.10-5.10; $\mathrm{P}=0.028)$. In the multivariate analysis, weight-bearing bone (WBB) was a prognostic factor for BM-OS time (HR, 6.29; CI 1.27-31.1; P=0.024). The shorter the duration and the higher the frequency of the Bps administration, the longer the BM-OS time (HR, 0.98; CI, 0.97-0.99; $\mathrm{P}<0.001$ and HR, 0.12; 0.04-0.32; $\mathrm{P}<0.001)$. Age was also a favorable prognostic factor for BaPFS in multivariate analyses ( $\mathrm{HR}, 0.96 ; 0.93-0.99 ; \mathrm{P}=0.014)$, while being female was a poor prognostic factor for BaPFS time (HR, 2.55; 1.31-4.97; $\mathrm{P}=0.006)$.

\section{Discussion}

EGFR mutation and ALK rearrangements are two kinds of genetic mutation discovered in NSCLC. About 5\% NSCLC patients harbor ALK rearrangements (17). Sensitive EGFR mutations are present in $\sim 15$ and $50 \%$ of lung adenocarcinoma cases in the Caucasian and Asian population, respectively (18). EGFR TKIs include gefitinib, erlotinib and icotinib. Crizotinib is the first-generation ALK TKI. Clinical trials have revealed that TKIs therapy results in longer PFS time, improved health-related quality of life and decreased treatment-related severe side effects in patients with sensitive mutations when compared with that in standard chemotherapy. Thus, many clinical guidelines recommend that all patients with sensitive
EGFR mutations or ALK rearrangements receive first-line treatment with TKIs in advanced NSCLC $(14,19)$.

The present study demonstrated that patients with NSCLC exhibiting BM had significantly prolonged PFS time in the Bps group compared with the non-Bps group. Among patients with genetic mutations who underwent TKI treatment (EGFR TKIs or ALK TKIs), the group that received combination therapy with Bps experienced significantly longer PFS time and tended to exhibit significantly prolonged BM-OS time compared with the non-Bps group. In the multivariate analysis, WBB was a poor prognostic marker for BM-OS time, where the shorter the duration and the greater the frequency of Bps administration, the longer the BM-OS time. Being female was also observed to be a better prognostic factor than being male for BM-OS time, while being male was associated with poor prognosis with respect to BaPFS time.

Bps can prevent and treat SAEs, improve the prognosis of patients with BM, and improve quality of life. The present study demonstrated that combination therapy with Bps significantly improved PFS time and improved BM-OS time by almost 6 months in comparison with non-Bps treatment in patients with NSCLC exhibiting BM. However, the two groups exhibited approximately the same BaPFS time. A retrospective study of 62 patients with NSCLC exhibiting BM demonstrated that, in comparison with the EGFR TKI alone regimen group, the EGFR TKI plus Bps group exhibited significantly 


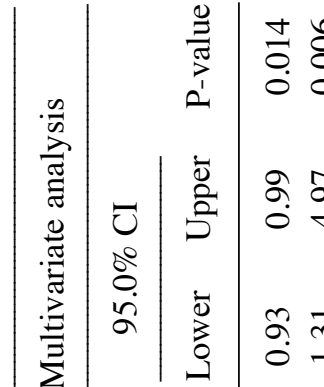

艾

苛

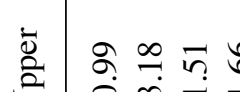

के लำ

告

ก

กู่

으 윰

ชై $\bar{\sigma}$

nิ กิ

列

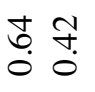

음

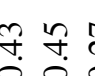

กิ้ กิ

त̂े

ㅇํㅇ ๖웅

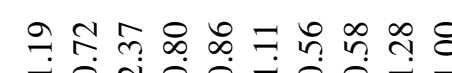

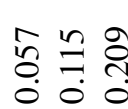

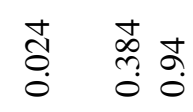

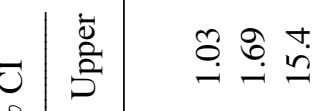

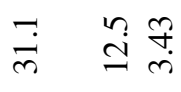

กิ

$\begin{array}{ccc}\infty & \infty & n \\ 0 & \infty & n \\ 0 & 0 & 0\end{array}$

กิ

तु $气 \stackrel{2}{i}$

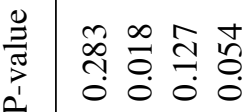

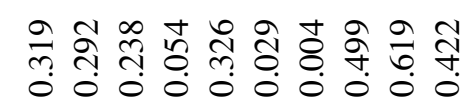

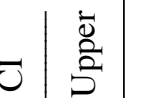

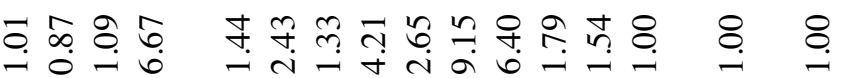

م.

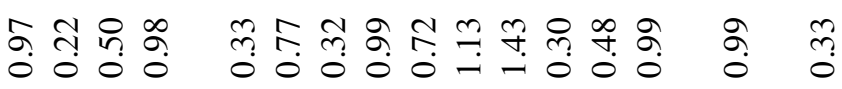

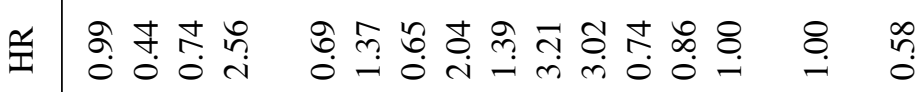

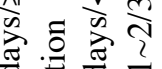

응 
prolonged PFS and OS time (mPFS: 15.0 vs. 7.3 months; $\mathrm{P}=00017$ and mOS: 25.2 vs. 10.4 months; $\mathrm{P}=0.0015$ ) (12). However, another retrospective study involving 356 patients with NSCLC exhibiting BM demonstrated that the EGFR-TKI plus Bps group had a statistically significantly longer PFS time (PFS: 11.6 vs. 9.3 months; HR, 0.68; $\mathrm{P}=0.009$ ) but similar OS time (OS: 20.5 vs. 19.5 months; HR, 0.95; $\mathrm{P}=0.743$ ) in patients with EGFR-mutant NSCLC and BM (13). As in the two aforementioned retrospective studies, the present study also demonstrated that in the genetic mutation group, Bps could enhance the antitumor effect of TKIs (including EGFR TKIs and ALK TKIs) to prolong PFS time in patients with NSCLC exhibiting BM, and numerically better BM-OS time was also observed. However, regardless of the genetic mutations, patients receiving Bps that also received TKIs regimen or EGFR TKIs regimen had significantly prolonged BM-OS time compared with TKIs alone or the EGFR TKIs alone regimen. It was the two patients without genetic mutations but received TKIs with Bps that made a difference. Considering the two patients were only tested for the EGFR gene mutation using PCR, and had better OS time after EGFR TKI regimen, we hypothesize that the two patients had an undetected EGFR mutation due to technical limitations and unknown mutation sites at the time of testing. As there were only two wild-type patients receiving TKI plus Bps therapy in the present study, conclusions regarding efficacy of TKI plus Bps therapy may not be accurately drawn in wild-type patients. Further studies are required in order to overcome this issue. Patients received chemotherapy after their diseases progressed on TKI treatment. As our study is retrospective, it's hard for us to draw a conclusion on whether chemotherapy makes a difference on survival. But previous published evidence revealed that there are no statistically significant differences in OS time between the third-generation chemotherapy regimens (20). In conclusion, our study found that addition of Bps to the TKI treatment regimen had a positive effect on survival outcomes for patients with NSCLC.

Considering the small sample size used in the present study, subsequent studies should be performed with larger sample sizes in order to eliminate errors. Studies have demonstrated that Bps can enhance the antitumor effect of EGFR TKIs in EGFR mutant tumor cells in vitro $(21,22)$. TKIs have been demonstrated to affect the activation of key cell signaling pathways, as gefitinib has been observed to inhibit the phosphorylation of EGFR-activated ERK1/2 and Akt. However, Bps has no known pharmacodynamic effect on the phosphorylation of ERK1/2 and Akt, but can block the Ras signaling pathway and subsequently induce cancer cell apoptosis (21). Another study also revealed that gefitinib combined with Bps could induce cancer cell apoptosis and regulate the production of mitotic and angiogenic factors (22). In vivo studies have demonstrated that erlotinib plus Bps significantly inhibited tumor growth over erlotinib alone in an EGFR-mutated NSCLC nude mouse tumor model, which indicated that Bps enhanced the antitumor efficacy of erlotinib. The combination also resulted in greater apoptosis by inhibiting Akt phosphorylation (23). The results indicated that the mechanistic basis of Bps-enhanced TKI antitumor activity may be associated with Bps-intrinsic antitumor properties and the decrease in adverse reactions from targeted therapy. The present study indicated that Bps may prolong the PFS time and trend to improve BM-OS time of patients with NSCLC exhibiting BM by enhancing the antitumor effect of TKIs, suggesting that patients with NSCLC harboring genetic mutations and also exhibiting BM treated with frontline TKIs (EGFR TKIs or ALK TKIs), should also be treated with Bps simultaneously.

Bps, however, demonstrated no benefit for BaPFS time in the present study, which may be explained by the small sample size. Bone formation markers, which included C-terminal telopeptides of type I collagen (ICTP), total alkaline phosphatase (tALP), bone-specific alkaline phosphatase (bALP), amino-terminal procollagen propeptides of type I collagen (PINP), cross-linked N-terminal (NTx) and cross-linked C-terminal, were of more diagnostic and prognostic significance than resorption marker in bone metabolism and survival in patients with metastatic prostate cancer (24). Similarly, the aforementioned bone markers were also significantly increased in patients with lung cancer exhibiting BM (25). A meta-analysis involving 3,268 patients with solid tumors demonstrated that serum bALP was significantly higher than in those patients without bone lesions (26). Another meta-analysis that included 1,279 patients, demonstrated a significant association between serum NTX and bone involvement (27). Hence, bone markers have been screened as diagnostic and prognostic markers, as well as for the development of anticancer drugs (28). As demonstrated in a previous study, serum concentration of the majority of bone markers including tALP, bALP and PINP, but not ICTP, were decreased following the administration of zoledronic acid (15). In particular, an investigational analysis was performed on 1,126 patients with breast cancer, castration-refractory prostate cancer, NSCLC and a variety of other solid tumors from three randomized trials regarding therapeutic effect of zoledronate acid vs. placebo. It was revealed that zoledronic acid significantly improved OS time in patients with elevated baseline NTX $(\geq 100 \mathrm{nmol} / \mathrm{mmol}$ creatinine; relative risks, 0.692; $\mathrm{P}=0.0028)(29)$, which indicated that patients with aggressive skeletal disease significantly benefited from zoledronate treatment. In the present study, no significant difference regarding BaPFS time was observed between the Bps group and non-Bps group or any other subgroups. Despite the advanced development on bone scan technique, BM detection often occurs in the symptomatic stage of disease, while serum bone markers changed ahead of the image examinations. The univariate analysis demonstrated that ALP was a predictive marker for NSCLC with BM. As a bone scan may not reflect the timely progression of BM, the need for novel strategies aimed at the early identification and detection of $\mathrm{BM}$ progression is urgent. Bone markers are the better choice, which can even total take the place of bone scan in the detection of BM progression for selected population (30).

In the multivariate analysis, WBB was an adverse prognostic factor for BM-OS time. Patients experienced longer BM-OS time when Bps was administered more frequently and over a shorter period of time. As weight-bearing BM was a poor prognostic indicator of BM-OS time, serious consideration should be given to the inclusion of Bps in the treatment plan for patients with weight-bearing BM. Being female was a favorable prognostic marker for BM-OS time, but a poor prognostic marker for BaPFS time. Zoledronic acid has been demonstrated to consistently improve disease-free 
survival in patients with breast cancer (31). However, this effect was limited to post-menopausal women in a number of trials $(32,33)$. In the clinical trial AZURE (34), Bps was demonstrated to effectively prevent local or non-BM recurrence in post-menopausal women. However, pre-menopausal women did not benefit from Bps treatment. A similar effect was observed in goserelin-treated patients in the ABCSG-12 study (33) Patients who received anastrozole or tamoxifen prior to the initiation of Bps treatment exhibited a good prognosis (33). This effect may be explained by the ability of estrogen to promote tumor angiogenesis, tumor invasion and metastasis (35). In the AZURE study, the premenopausal patients demonstrated an unfavorable prognosis, with $>95 \%$ patients requiring further chemotherapy (34). In contrast, menopausal patients in the ABCSG-12 study exhibited a better prognosis, with $<5 \%$ patients receiving chemotherapy as all the rest responded well to anti-estrogen therapy prior to the initiation of Bps treatment. Based on the finding that being female was an unfavorable predictor for BaPFS time in the present study, it can be assumed that inhibition of estrogen could be a feasible way to improve prognosis. However, it was also revealed that female patients had improved BM-OS time than male patients. It is well known that estrogen could decrease the expression of RANKL, which promoted osteolysis via binding with RANK on osteoclasts (36). Whereas, zoledronic acid executed its anticancer function primarily through the inhibition of osteoclastic bone resorption, which is more prominent in the absence of estrogen (37). These observations can explain the opposite role of estrogen revealed by the previous studies. However, how estrogen promoted the metastasis in the bone and prolonged OS time under treatment of zoledronic acid remains unclear, and further research is required in order to elucidate this query.

The present study had some limitations. First, although the baseline population was large, there was only a small number of patients who fulfilled the eligibility criteria of the present study. In addition, patients in the subgroups had small-scale cancer, and certain patients lacked basic information, which may have led to errors in the results. A larger cohort study should be performed in order to confirm these findings. Secondly, the time to receive Bps and the duration and frequency of Bps treatment varied greatly between patients, which may have caused huge individual differences. Thirdly, as the present study was retrospective in design, factors such as the diverse treatment regimens, and uncertainty about the exact time of death, may confound the results of the study.

The results of the present study revealed that in TKI-treated patients with NSCLC harboring genetic mutations, those who also received Bps had significantly longer PFS time in comparison with those patients who did not receive Bps and tended to improve BM-OS time. These findings indicate that patients with NSCLC harboring genetic mutations who also exhibited BM and were being treated with frontline TKIs, or any patient in need of subsequent treatment, should be treated with Bps simultaneously. However, the present study is limited by its retrospective design, and therefore may be subject to errors due to its small sample size. Large-scale randomized trials with rigorously controlled treatment regimens should be performed in the future in order to identify the functional impact of Bps on clinical outcomes of patients with NSCLC patients exhibiting BM.

\section{Acknowledgements}

Not applicable.

\section{Funding}

The present study was supported by grants from the National Natural Science foundation of China (grant no. 81570008) and the Medical Scientific Research Foundation of Guangdong Province of China (grant no. A2015311).

\section{Availability of data and materials}

All data generated or analyzed during this study are included in this published article.

\section{Authors' contributions}

$\mathrm{XC}$ and $\mathrm{YZ}$, contributed to the conception and design of the study. YZ provided administrative support. ZL, BPL, LH, JF and BML contributed to collection and assembly of data. XC, SL and JG performed data analysis and interpretation. All authors were involved in the writing of the manuscript and all authors give final approval of manuscript for publication.

\section{Ethics approval and consent to participate}

The present study was approved by the Ethics Committee of The First Affiliated Hospital of Sun Yat-sen University. A waiver was granted excusing the requirement of written informed consent due to the retrospective nature of the present study.

\section{Patient consent for publication}

Not applicable.

\section{Competing interests}

The authors have declared no competing interests.

\section{References}

1. Chen W, Zheng R, Baade PD, Zhang S, Zeng H, Bray F, Jemal A, Yu XQ and He J: Cancer statistics in China, 2015. CA Cancer J Clin 66: 115-132, 2016.

2. Novello S, Barlesi F, Califano R, Cufer T, Ekman S, Levra MG Kerr K, Popat S, Reck M, Senan S, et al: Metastatic non-small-cell lung cancer: ESMO Clinical Practice Guidelines for diagnosis, treatment and follow-up. Ann Oncol 27 (Suppl 5): v1-v27, 2016.

3. Stinchcombe TE, Lee CB and Socinski MA: Current approaches to advanced-stage non-small-cell lung cancer: First-line therapy in patients with a good functional status. Clin Lung Cancer 7 (Suppl 4): S111-S117, 2006.

4. Coleman RE: Metastatic bone disease: Clinical features, pathophysiology and treatment strategies. Cancer Treat Rev 27: 165-176, 2001.

5. Brodowicz T, O'Byrne $\mathrm{K}$ and Manegold C: Bone matters in lung cancer. Ann Oncol 23: 2215-2222, 2012.

6. Coello MC, Luketich JD, Litle VR and Godfrey TE: Prognostic significance of micrometastasis in non-small-cell lung cancer. Clin Lung Cancer 5: 214-225, 2004.

7. Tsuya A and Fukuoka M: Bone metastases in lung cancer. Clin Calcium 18: 455-459, 2008 (In Japanese). 
8. Dhillon S: Zoledronic Acid $(\operatorname{Reclast}((\mathrm{R}))$, Aclasta((R))): A Review in Osteoporosis. Drugs 76: 1683-1697, 2016.

9. Dunford JE, Thompson K, Coxon FP, Luckman SP, Hahn FM, Poulter CD, Ebetino FH and Rogers MJ: Structure-activity relationships for inhibition of farnesyl diphosphate synthase in vitro and inhibition of bone resorption in vivo by nitrogen-containing bisphosphonates. J Pharmacol Exp Ther 296: 235-242, 2001.

10. Li YY, Chang JW, Chou WC, Liaw CC, Wang HM, Huang JS Wang $\mathrm{CH}$ and Yeh KY: Zoledronic acid is unable to induce apoptosis, but slows tumor growth and prolongs survival for non-small-cell lung cancers. Lung Cancer 59: 180-191, 2008.

11. Lu S, Zhang J, Zhou Z, Liao ML, He WZ, Zhou XY, Li ZM, Xiang JQ, Wang JJ and Chen HQ: Synergistic inhibitory activity of zoledronate and paclitaxel on bone metastasis in nude mice. Oncol Rep 20: 581-587, 2008.

12. Huang CY, Wang L, Feng CJ, Yu P, Cai XH, Yao WX, Xu Y, Liu XK Zhu WJ, Wang Y, et al: Bisphosphonates enhance EGFR-TKIs efficacy in advanced NSCLC patients with EGFR activating mutation: A retrospective study. Oncotarget 7: 66480-66490, 2016.

13. Zhang G, Cheng R, Zhang Z, Jiang T, Ren S, Ma Z, Zhao S, Zhou $\mathrm{C}$ and Zhang $\mathrm{J}$ : Bisphosphonates enhance antitumor effect of EGFR-TKIs in patients with advanced EGFR mutant NSCLC and bone metastases. Sci Rep 7: 42979, 2017.

14. Ettinger DS, Wood DE, Aisner DL, Akerley W, Bauman J, Chirieac LR, D'Amico TA, DeCamp MM, Dilling TJ, Dobelbower M, et al: Non-small cell lung cancer, version 5.2017, NCCN clinical practice guidelines in oncology. J Natl Compr Canc Netw 15: 504-535, 2017.

15. Lein M, Wirth M, Miller K, Eickenberg HU, Weissbach L, Schmidt K, Haus U, Stephan C, Meissner S, Loening SA and Jung K: Serial markers of bone turnover in men with metastatic prostate cancer treated with zoledronic Acid for detection of bone metastases progression. Eur Urol 52: 1381-1387, 2007.

16. Eisenhauer EA, Therasse P, Bogaerts J, Schwartz LH, Sargent D, Ford R, Dancey J, Arbuck S, Gwyther S, Mooney M, et al: New response evaluation criteria in solid tumours: Revised RECIST guideline (version 1.1). Eur J Cancer 45: 228-247, 2009.

17. Chia PL, Mitchell P, Dobrovic A and John T: Prevalence and natural history of ALK positive non-small-cell lung cancer and the clinical impact of targeted therapy with ALK inhibitors. Clin Epidemiol 6: 423-432, 2014.

18. Midha A, Dearden S and McCormack R: EGFR mutation incidence in non-small-cell lung cancer of adenocarcinoma histology: A systematic review and global map by ethnicity (mutMapII). Am J Cancer Res 5: 2892-2911, 2015.

19. ESMO Guidelines Committee: Appendix 8: Metastatic non-small-cell lung cancer (2): eUpdate published online 28 June 2017 (www.esmo.org/Guidelines/Lung-and-Chest-Tumours). Ann Oncol 28 (Suppl_4): iv162-iv164, 2017.

20. Pilkington G, Boland A, Brown T, Oyee J, Bagust A and Dickson R: A systematic review of the clinical effectiveness of first-line chemotherapy for adult patients with locally advanced or metastatic non-small cell lung cancer. Thorax 70: 359-367, 2015.

21. Chang JW, Hsieh JJ, Shen YC, Yeh KY, Wang CH, Li YY and Hsu T: Bisphosphonate zoledronic acid enhances the inhibitory effects of gefitinib on EGFR-mutated non-small cell lung carcinoma cells. Cancer Lett 278: 17-26, 2009.

22. Melisi D, Caputo R, Damiano V, Bianco R, Veneziani BM, Bianco AR, De Placido S, Ciardiello F and Tortora G: Zoledronic acid cooperates with a cyclooxygenase-2 inhibitor and gefitinib in inhibiting breast and prostate cancer. Endocr Relat Cancer 12: 1051-1058, 2005.

23. Stachnik A, Yuen T, Iqbal J, Sgobba M, Gupta Y, Lu P,Colaianni G, Ji Y, Zhu LL, Kim SM, et al: Repurposing of bisphosphonates for the prevention and therapy of nonsmall cell lung and breast cancer. Proc Natl Acad Sci USA 111: 17995-18000, 2014.
24. Cook RJ, Coleman R, Brown J, Lipton A, Major P, Hei YJ, Saad F and Smith MR: Markers of bone metabolism and survival in men with hormone-refractory metastatic prostate cancer. Clin Cancer Res 12: 3361-3367, 2006.

25. Aruga A, Koizumi M, Hotta R, Takahashi S and Ogata E: Usefulness of bone metabolic markers in the diagnosis and follow-up of bone metastasis from lung cancer. Br J Cancer 76: 760-764, 1997.

26. Du WX, Duan SF, Chen JJ, Huang JF, Yin LM and Tong PJ: Serum bone-specific alkaline phosphatase as a biomarker for osseous metastases in patients with malignant carcinomas: A systematic review and meta-analysis. J Cancer Res Ther 10 (Suppl): C140-C143, 2014

27. Zhang Y, Yi M, Cao J, Hou C, Zhou Y and Zhong Y: Serum cross-linked N-telopeptide of type I collagen for the diagnosis of bone metastases from solid tumours in the Chinese population: Meta-analysis. J Int Med Res 44: 192-200, 2016.

28. D'Oronzo S, Brown J and Coleman R: The role of biomarkers in the management of bone-homing malignancies. J Bone Oncol 9: $1-9,2017$.

29. Coleman RE, Lipton A, Costa L, Cook RJ, Lee KA, Saad F, Brown JE, Terpos E, Major PP, Kohno N, et al: Possible survival benefits from zoledronic acid treatment in patients with bone metastases from solid tumours and poor prognostic features-An exploratory analysis of placebo-controlled trials. J Bone Oncol 2: 70-76, 2013.

30. Ferreira A, Alho I, Casimiro S and Costa L: Bone remodeling markers and bone metastases: From cancer research to clinical implications. Bonekey Rep 4: 668, 2015.

31. Aft RL, Naughton M, Trinkaus K and Weilbaecher K: Effect of (Neo)adjuvant zoledronic acid on disease-free and overall survival in clinical stage II/III breast cancer. Br J Cancer 107: $7-11,2012$.

32. Coleman R, de Boer R, Eidtmann H, Llombart A, Davidson N, Neven $P$, von Minckwitz G, Sleeboom HP, Forbes J, Barrios C, et al: Zoledronic acid (zoledronate) for postmenopausal women with early breast cancer receiving adjuvant letrozole (ZO-FAST study): Final 60-month results. Ann Oncol 24: 398-405, 2013

33. Gnant M, Mlineritsch B, Stoeger H, Luschin-Ebengreuth G, Heck D, Menzel C, Jakesz R, Seifert M, Hubalek M, Pristauz G, et al: Adjuvant endocrine therapy plus zoledronic acid in premenopausal women with early-stage breast cancer: 62-month follow-up from the ABCSG-12 randomised trial. Lancet Oncol 12: 631-641, 2011.

34. Coleman R, Cameron D, Dodwell D, Bell R, Wilson C, Rathbone E, Keane M, Gil M, Burkinshaw R, Grieve R, et al: Adjuvant zoledronic acid in patients with early breast cancer: final efficacy analysis of the AZURE (BIG 01/04) randomised open-label phase 3 trial. Lancet Oncol 15: 997-1006, 2014.

35. Steinman RA, Brufsky AM and Oesterreich S: Zoledronic acid effectiveness against breast cancer metastases-a role for estrogen in the microenvironment? Breast Cancer Res 14: 213, 2012.

36. Frenkel B, Hong A, Baniwal SK, Coetzee GA, Ohlsson C, Khalid $\mathrm{O}$ and Gabet Y: Regulation of adult bone turnover by sex steroids. J Cell Physiol 224: 305-310, 2010.

37. Wright LE and Guise TA: The microenvironment matters: Estrogen deficiency fuels cancer bone metastases. Clin Cancer Res 20: 2817-2819, 2014.

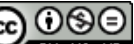

This work is licensed under a Creative Commons Attribution-NonCommercial-NoDerivatives 4.0 International (CC BY-NC-ND 4.0) License. 